\title{
OBJECT-BASED IMAGE ENHANCEMENT TECHNIQUE FOR GRAY SCALE IMAGES
}

\author{
G. Srinivasa Rao ${ }^{1}$ Dr. A. Sri Krishna ${ }^{2}$ Dr. S. Mahaboob Basha ${ }^{3}$ Ch. Jeevan \\ Prakash $^{4}$ \\ Department of Information Technology, R.V.R \& J.C College of Engineering, \\ Guntur, Andhra Pradesh
}

\begin{abstract}
Image enhancement technique plays vital role in improving the quality of the image. Enhancement technique basically enhances the foreground information and retains the background and improve the overall contrast of an image. In some case the background of an image hides the structural information of an image. This paper proposes an algorithm which enhances the foreground image and the background part separately and stretch the contrast of an image at inter-object level and intra-object level and then combines it to an enhanced image. The results are compared with various classical methods using image quality measures.
\end{abstract}

\section{KEYWORDS}

Image Enhancement, Morphological watershed segmentation, Object-based contrast enhancement, Interobject stretching, Intra-object stretching.

\section{INTRODUCTION}

Basically image enhancement [3] improves the quality of the image so that the result is more suitable for a specific application and for human perception. Image enhancement techniques are widely used in many real time applications. The contrast enhancement in digital images can be handled by using various point processing techniques[2]-[7] like power law, logarithmic transformations and histogram equalization(HE).Image enhancement using power law transformations depends upon the gamma values, if the gamma value exceeds 1 , the contrast is reduced.The logarithmic transformation [2]-[4] improve the contrast of the image, but increases the overall brightness.The most widely used technique of Contrast enhancement is Histogram Equalization (HE)[1]-[5], which works by flattening the histogram and stretching the dynamic range of the gray-levels using the cumulative density function of the image. However, there are some drawbacks with histogram equalization [8] especially when implemented to process digital images. Firstly, it converts the histogram of the original image into a uniform histogram with a mean value at the middle of gray level range. So, the average intensity value of the output image is always at the middle - or close to it. In the case of images with high and low average intensity values, there is a significant change in the image outlook after enhancing the contrast and some noise is also introduced into the image. Secondly, histogram equalization enhances the image based on the global content of the image and in its discrete form large bins cannot be broken and reordered to produce the desired uniform histogram. In other words, HE is powerful in highlighting the borders and edges between different objects, but it may reduce the local details within these objects, particularly smooth and small ones. One more consequence for this

DOI : 10.5121/ijait.2014.4302 
mergence between large and small bins is the creation of over enhancement and saturation artifacts [10]. Recently a histogram equalization based contrast enhancement has been proposed [24], which improve the image in poor lightning. Many attempts have been made so far to improve the performance of Histogram Equalization [3],[8]-[10],[24].

Even though image enhancement techniques, generally not required for automated analysis techniques, have regained a significant interest in recent years. Most of the existing automatic enhancement techniques make use of global intensity transforms, either for color correction (white balancing) or contrast enhancement. For these global intensity transforms, the mapping of color or intensity is one-to-one and is independent of pixel location or scene context. Such techniques would not work well for images where different parts of the image require different types of correction, e.g., the darker portions of an indoor scene requires higher contrast tuning and different Color adjustment than the window, or it is necessary to highlight the subject by enhancing contrast between the subject and its background [1]. The Object Based Multi-Level Contrast Stretching Method dividing the input image into its constituent objects, and apply stretching strategies based on type of the objects. This object-based multilevel enhancement method can produce enhanced images without ringing, blocking and false contouring artifacts.

This paper proposes an object based technique to enhance the local contrast in the spatial domain. It uses morphological opening operation for finding gradient thresholding. The paper is organized as follows. Section 2 describes the Basic Terminology. Section 3 describes Object Based Contrast Stretching (OBCS). Section 4 presents the Comparison Of Object-Based Contrast Stretching with existing methods. Section 5 presents conclusions.

\section{BASIC TERMINOLOGY}

\subsection{Morphological OPERAtions}

Mathematical morphology [6] is the part of set theory, which has a robust geometric orientation. Mathematical morphology presents a well-found theory for analysis and processing for binary images.

Consider a $2 \mathrm{D}$ digital image $\mathrm{A} \subseteq \mathrm{Z} \times \mathrm{Z}$ and a point $\mathrm{u} \in \mathrm{Z} \times \mathrm{Z}$, the transition of $\mathrm{A}$ by $\mathrm{u}$ is given by equation (1)

$$
(A)=\{u+u \mid a \in A\}
$$

Dilation and Erosion are the fundamental morphological operations which are given by (2) and (3) respectively

$$
\begin{aligned}
\delta_{\mu B} A & =\mathrm{V}_{b \in B}(A)_{b} \\
\varepsilon_{\mu B} A & =\wedge_{b \in B}(A)_{b}
\end{aligned}
$$

Here $B$ is a structuring element.

In terms of dilation and erosion another pair of morphological operations are defined known as closing and opening given by (4) and (5) respectively.. 


$$
\begin{aligned}
& \varphi_{\mu B} A=\varepsilon_{\mu D}\left(\delta_{\mu D} A\right) \\
& \gamma_{\mu B} A=\delta_{\mu B}\left(\varepsilon_{\mu B} A\right)
\end{aligned}
$$

\subsection{EDGE DETECTION}

An edge [7] is a connected set of pixels on the boundary between two regions. There are many ways to perform edge detection. The edge detection is categorized into two categories.

1) The First Order Derivative.

2) Second Order Derivative.

The First Order Derivative is a gradient method which detects the edges by looking for the magnitude of gradient in the image, and the Laplacian method searches for zero crossings in the second derivative of the image is used to find edges.

The magnitude of the gradient $G(x, y)$ of the image $I(x, y)$ is defined as given in equation (6)

$G(x, y)=\left[\mathrm{G}_{\mathrm{X}}^{2}(\mathrm{x}, \mathrm{y})+\mathrm{G}_{\mathrm{Y}}^{2}(\mathrm{x}, \mathrm{y})\right]^{1 / 2}=\left[[\partial \mathrm{I}(\mathrm{x}, \mathrm{y}) / \partial \mathrm{x}]^{2}+[\partial \mathrm{I}(\mathrm{x}, \mathrm{y}) / \partial \mathrm{y}]^{2}\right]^{1 / 2}$

For the given input image an efficient gradient computation is applied to find the edges, i.e., smoothing, sharpening the image. The gradient components $\mathrm{G}_{\mathrm{X}}(\mathrm{x}, \mathrm{y})$ and $\mathrm{G}_{\mathrm{Y}}(\mathrm{x}, \mathrm{y})$ can be obtained by using the Prewit or Sobel operator as given in Fig 1. A more practical version of $G(x, y)$ is its approximation using absolute values as given in equation (7).

$$
G(x, y)=\left|G_{X}(\mathrm{x}, \mathrm{y})\right|+\left|\mathrm{G}_{\mathrm{Y}}(\mathrm{x}, \mathrm{y})\right|
$$

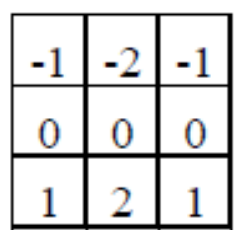

(a)

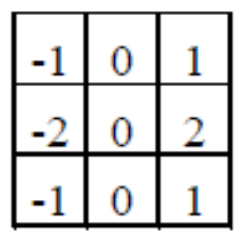

(b)

Fig.1: Sobel masks used to compute gradient components along $G_{X}$ and $G_{Y}$

\subsection{MORPHOLOGicAl WATERSHED}

The watershed transform [13]-[16] is a well known segmentation method coming from the field of mathematical morphology. Watershed algorithm for image segmentation on the grey levels of neighbouring pixels, assumes that a hole is drilled in minimum of the surface, and water flows from different positions into the hole. The hole is supposed to be a central pixel of a neighbourhood. For the following $3 \times 3$ and $5 \times 5$ grey level masks, the watersheds are recognized with a peak or highest level $\mathrm{h}>=20$, and the Peak points are shown in Fig 2 and Fig 3. 


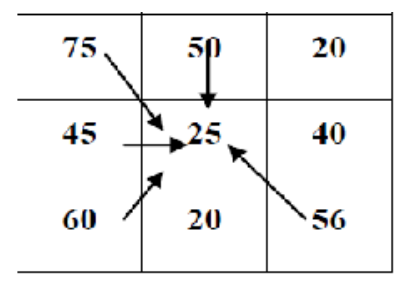

Fig. 2 Example of Grey level watershed with height $\mathrm{h}>=20$.

When we increase highest level (h) value, water will fall from peak top points to bottom points and creates a good watershed.

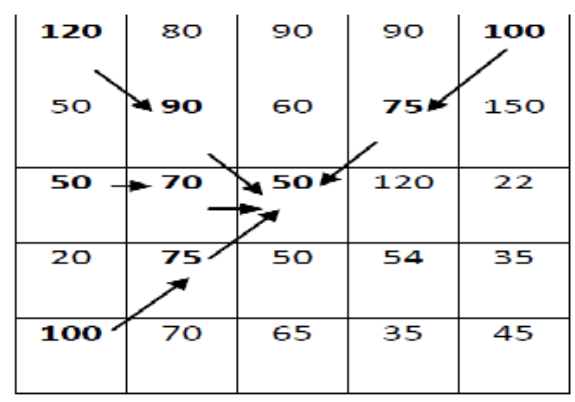

Fig. 3 Watershed of grey level Image with 5 X5 mask

\subsection{REGION GROWING}

Based on the similarity constraints, Region Growing [17]-[18] method merges small pixel regions or sub regions into larger regions. Identify the set of seed points in the image, and region growing is applied to each seed pixel and is appending with adjacent pixels that have similar properties like color or gray level value.

\section{ObJect BaSEd Contrast STRETChing}

The process of object based contrast stretching includes the pre-processing step by finding the edges of an image, and then the threshold is applied on the gradient image which is given as marker to the morphological watershed algorithm. The morphological watershed segments the image into various objects. As the watershed algorithm results in over segmentation, the region growing algorithm is applied for partitioning the image into two significant regions i.e. the foreground and background regions. The contrast stretching is separately applied to each region and after enhancement of each region, the regions are merged to form an enhanced image. The complete process of object based contrast stretching is given in Fig 4.

\subsection{Gradient Computation}

For the given input image efficient edge detection algorithm is applied using Sobel operator.

Step 1: Apply Sobel filter to the input image to find edges.

- In this step apply gradient computation on the input image using $\mathrm{G}_{\mathrm{X}}$ and $\mathrm{G}_{\mathrm{Y}}$ along $\mathrm{X}$-direction and Y-direction respectively. 
- Now calculate $\mathrm{G}(\mathrm{x}, \mathrm{y})$ using equation (7).

- The resultant image is a edge preserved image.

\section{Algorithm: Gradient_Computation (I)}

Input: I (original image)

Output: $\mathrm{G}$ (edge preserved image)

\{

$\%$ Apply sobel mask along $\mathrm{x}$-direction on image I

$\mathrm{G}_{\mathrm{x}}(\mathrm{x}, \mathrm{y})=\left|\left(\mathrm{z}_{7}+2 \mathrm{z}_{8}+\mathrm{z}_{9}\right)-\left(\mathrm{z}_{1}+2 \mathrm{z}_{2}+\mathrm{z}_{3}\right)\right|$

$\%$ Apply sobel mask along y-direction on image I

$\mathrm{G}_{\mathrm{y}}(\mathrm{x}, \mathrm{y})=\left|\left(\mathrm{z}_{3}+2 \mathrm{z}_{6}+\mathrm{z}_{9}\right)-\left(\mathrm{z}_{1}+2 \mathrm{z}_{4}+\mathrm{z}_{7}\right)\right|$

$\%$ Sum of the two filtered images is the edge preserved image.

$\mathrm{G}(\mathrm{x}, \mathrm{y}) \neq\left(\mathrm{z}_{7}+2 \mathrm{z}_{8}+\mathrm{z}_{9}\right)-\left(\mathrm{z}_{1}+2 \mathrm{z}_{2}+\mathrm{z}_{3}\right)|+|\left(\mathrm{z}_{3}+2 \mathrm{z}_{6}+\mathrm{z}_{9}\right)-\left(\mathrm{z}_{1}+2 \mathrm{z}_{4}+\mathrm{z}_{7}\right) \mid$

\}

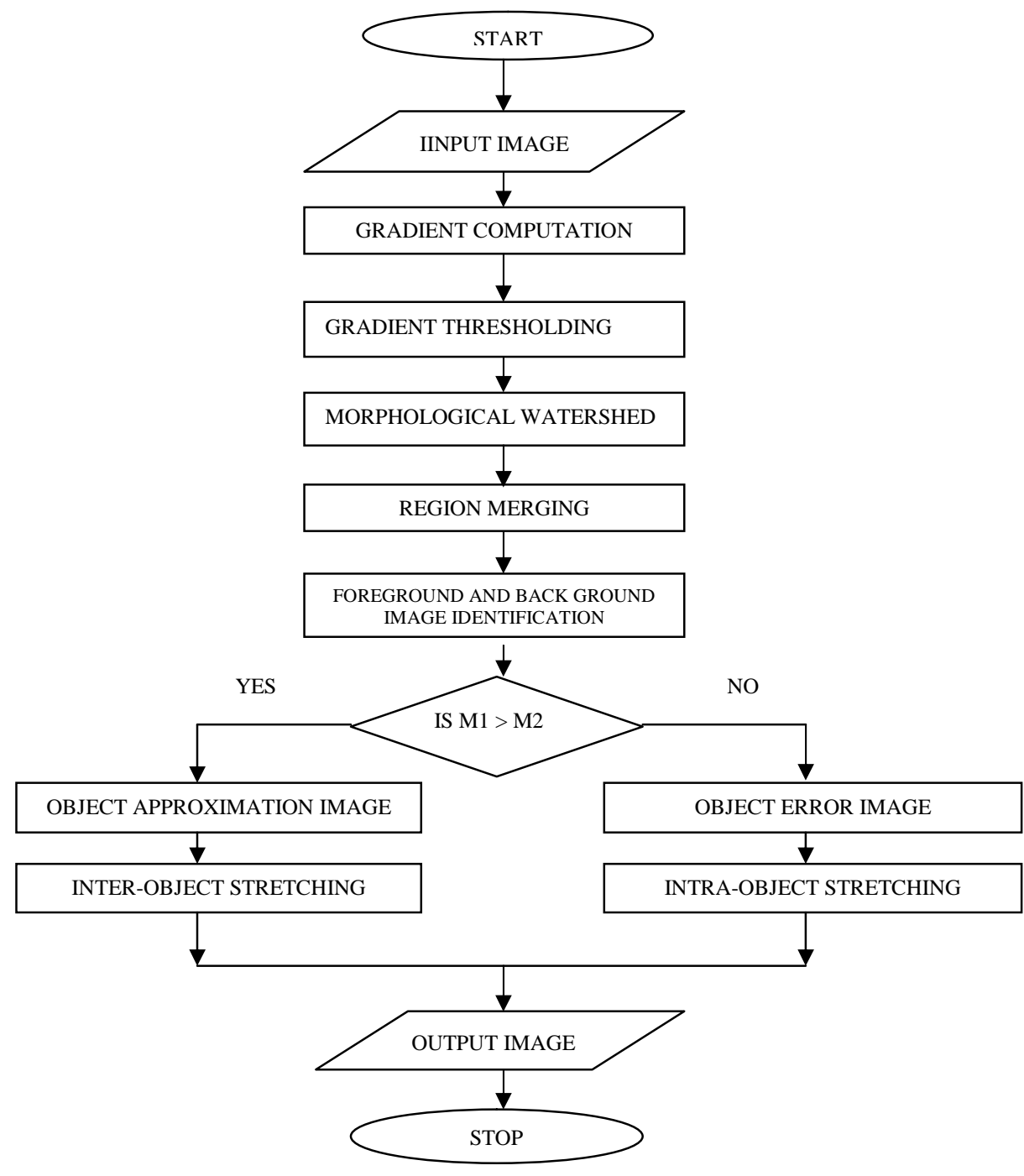

Fig. 4 Flow chart of Proposed Method 


\section{Algorithm: Gradient_Computation (I)}

Input: I (original image)

Output: G (edge preserved image) \{

$\%$ Apply sobel mask along $\mathrm{x}$-direction on image I

$\mathrm{G}_{\mathrm{x}}(\mathrm{x}, \mathrm{y})=\left|\left(\mathrm{z}_{7}+2 \mathrm{z}_{8}+\mathrm{z}_{9}\right)-\left(\mathrm{z}_{1}+2 \mathrm{z}_{2}+\mathrm{z}_{3}\right)\right|$

$\%$ Apply sobel mask along y-direction on image I

$\mathrm{G}_{\mathrm{y}}(\mathrm{x}, \mathrm{y})=\left|\left(\mathrm{z}_{3}+2 \mathrm{z}_{6}+\mathrm{z}_{9}\right)-\left(\mathrm{z}_{1}+2 \mathrm{z}_{4}+\mathrm{z}_{7}\right)\right|$

$\%$ Sum of the two filtered images is the edge preserved image.

$\mathrm{G}(\mathrm{x}, \mathrm{y}) \neq\left(\mathrm{z}_{7}+2 \mathrm{z}_{8}+\mathrm{z}_{9}\right)-\left(\mathrm{z}_{1}+2 \mathrm{z}_{2}+\mathrm{z}_{3}\right)|+|\left(\mathrm{z}_{3}+2 \mathrm{z}_{6}+\mathrm{z}_{9}\right)-\left(\mathrm{z}_{1}+2 \mathrm{z}_{4}+\mathrm{z}_{7}\right) \mid$

\}

\subsection{Gradient Thresholding}

The approach of gradient thresholding provides a simple and effective way to prevent oversegmentation, by providing markers to watershed segmentation. The process is defined by equation (8).

$$
G_{t h}(x, y)=\left\{\begin{array}{lr}
G_{T} & \text { if } G(x, y)<G_{T} \\
G(x, y) & \text { otherwise }
\end{array}\right.
$$

where $G_{t h}(x, y)$ is the threshold gradient magnitude, $G_{T}$ is a given threshold. The value of $G_{T}$ can be determined based on the following condition.

$$
\mathrm{G}_{\mathrm{T}}(\mathrm{x}, \mathrm{y})=(\mathrm{G} \text { o } \mathrm{B})(\mathrm{x}, \mathrm{y})+[\mathrm{G}(\mathrm{x}, \mathrm{y})-(\mathrm{G} \text { o } \mathrm{B})(\mathrm{x}, \mathrm{y})]
$$

Where $\mathrm{G}$ o $\mathrm{B}$ is the opening operation performed on $\mathrm{G}$ by the disk shape structuring element $\mathrm{B}$ of size $3 * 3$.

For the given input image an efficient gradient thresholding is applied to find the improved threshold image.

Step 2: Apply Gradient Thresholding to the Gradient Computation image to find improved threshold image.

- In this step apply open operation to the Gradient Computation image.

- Now calculate $\mathrm{G}_{\mathrm{T}}(\mathrm{x}, \mathrm{y})$ using equation (9).

- Now find the $\mathrm{G}_{\mathrm{th}}(\mathrm{x}, \mathrm{y})$ using equation (8), and the resultant image is an efficient gradient threshold image.

\section{Algorithm: Gradient_Thresholding (G)}

Input: $\mathrm{G}$ (edge image) 
Output: $\mathrm{G}_{\mathrm{th}}$ (improved threshold image) \{

$\%$ Apply open filter on image $\mathrm{G}$

$\mathrm{G} \circ \mathrm{B}=\delta \mathrm{B}(\varepsilon \mathrm{B}(\mathrm{G}))$

$\% \delta \mathrm{B}=$ erosion with $\mathrm{B}$ on $\mathrm{G}$

$\% \varepsilon \mathrm{B}=$ dilation with $\mathrm{B}$ on $\mathrm{G}$

$\%$ Calculate the threshold $\mathrm{G}_{\mathrm{T}}$

$\mathrm{G}_{\mathrm{T}}(\mathrm{x}, \mathrm{y})=(\mathrm{G}$ o $\mathrm{B})(\mathrm{x}, \mathrm{y})+[\mathrm{G}(\mathrm{x}, \mathrm{y})-(\mathrm{G}$ o $\mathrm{B})(\mathrm{x}, \mathrm{y})]$

$\%$ Find the gradient thresholding $\mathrm{G}_{\mathrm{th}}(\mathrm{x}, \mathrm{y})$ which is the improved gradient threshold image \}

\subsection{Segmentation Using Watersheds}

Step 3: Apply Morphological Watershed to the improved gradient threshold image to get the segmented image.

- This step applies watershed operation to the Gradient Thresholding image.

- The resultant image is a over segmented image.

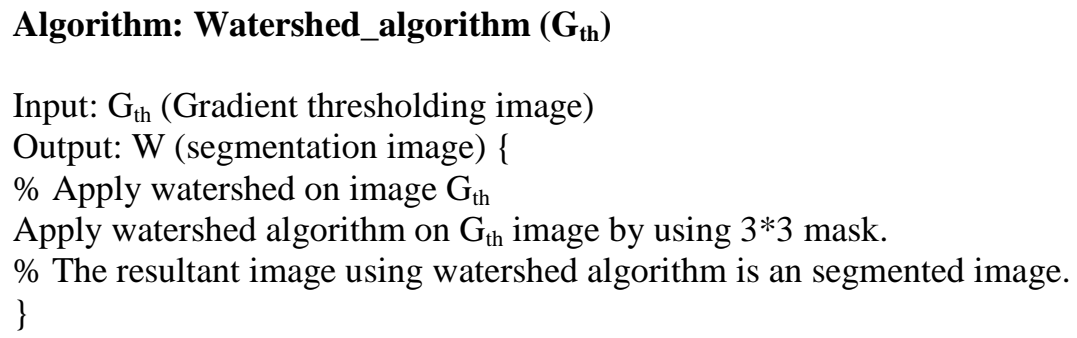

\subsection{Region Growing}

Step 4: Apply Region Growing to the watershed segmentation image.

- In this step apply Region Growing operation to the watershed segmentation image.

- The resultant image is a partitioned into two regions.

\section{Algorithm: Region_Growing (W)}

Input: W (watershed image)

Output: R (partitioned image) \{

$\%$ Apply Region Growing on image W

Select a seed pixel and grow the region until terminating condition is reached.

$\%$ The resultant image using Region Growing is Region partitioned image. \}

\subsection{FOREGROUND AND BACKGROUND IDENTIFICATION}

After image segmentation the segmented regions featuring homogeneous intensity and bearing contrast to their adjacent neighbours are extracted. These regions are treated as the objects that constitute the image. In order to enhance the image at inter-object level and intra-object level 
respectively, the image is split into two sub-images namely Object approximation image and Object error image.

Step 5: In this step the the Foreground and Background images are detected.

$$
\begin{aligned}
M 1= & R 1(i, j) \\
M 2= & R 2(i, j) \\
& \text { If }(M 1>M 2) \\
& R 1 \text { is the foreground image } \\
& \text { Else } \\
& R 1 \text { is the background image }
\end{aligned}
$$

\subsection{Object Approximation Image}

The object approximation image $\left(\mathrm{I}_{\mathrm{a}}\right)$ can be obtained by subtracting the mean value of watershed image $(\mathrm{W})$ of the corresponding region from the input image(I).

Step 6: In this step subtract mean value of the watershed image of the corresponding region from each pixel intensity value of the input image to get object approximation image.

\section{Algorithm: Approximation_Image (I, R)}

Input: I(Input Image), R(partitioned Image) OutputC (Object Approximation Image) \{

$\%$ calculate the mean values of both regions from partitioned image and find the object approximation image using equation (10).

\subsection{Object Error Image}

The Object Error Image is obtained by using the expression

$$
\mathrm{I}_{\varepsilon}(\mathrm{x}, \mathrm{y})=\mathrm{I}_{\mu}(\mathrm{x}, \mathrm{y})-\mathrm{I}_{\mathrm{a}}(\mathrm{x}, \mathrm{y})
$$

Where $I_{a}(x, y)$ is an Object Approximation Image, $I_{\varepsilon}(x, y)$ is an Object Error Image and $I_{\mu}(x, y)$ is an Mean value of the original input image.

Step 7: In this step subtract the value of approximation image from the mean value of the input image to get object error image.

\section{Algorithm: Error_Image (I, $\left.\mathbf{I}_{\mathbf{a}}\right)$}

Input: $\mathrm{I}\left(\right.$ Input Image), $\mathrm{I}_{\mathrm{a}}$ (object approximation image)

Output: $I_{\varepsilon}$ (Object Error Image $)\{$

$\%$ calculate the mean value of the input image

$\%$ each pixel intensity value of approximation image is subtracted from mean of the input image to get the resultant error image

\} 


\subsection{Object Contrast Stretching}

The contrast stretching is applied on the image objects at inter object level and intra object level. At inter object level an approach of stretching between adjacent local extremes is used to change the local contrast between objects. At intra object level the uniform linear stretching is used to enhance the textural features of objects while maintaining their homogeneity.

\subsubsection{Inter Object Stretching}

Step 8: In this step stretching between adjacent local extremes is used to change the local contrast between objects.

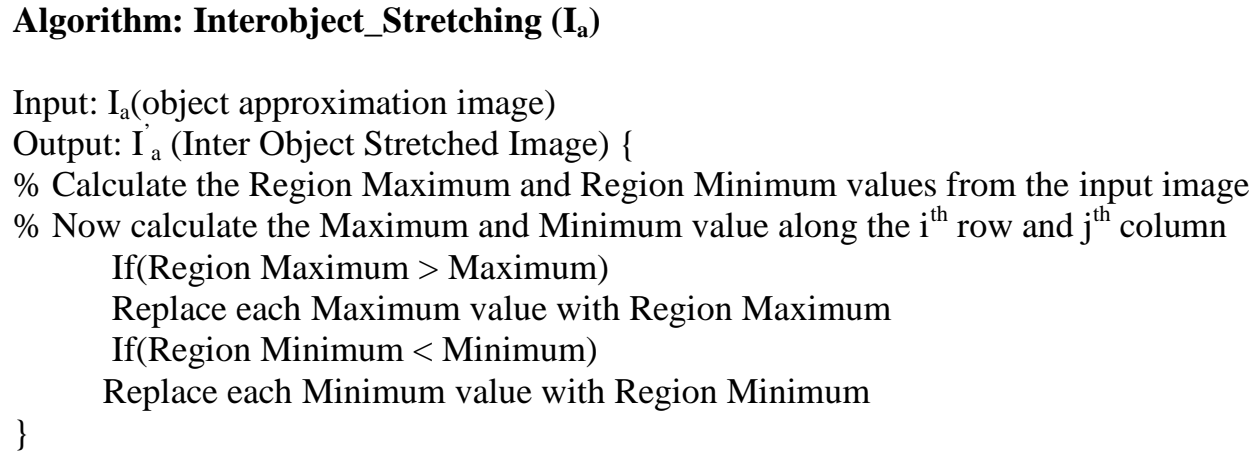

\subsubsection{Intra Object Stretching}

Step 9: In this step the uniform linear stretching is used to enhance the textural features of objects while maintaining their homogeneity.

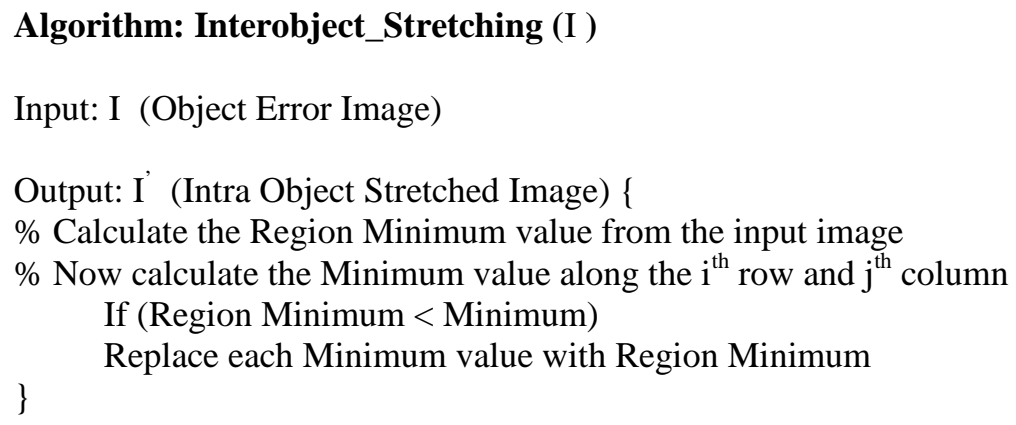

\subsection{Output Enhanced Image}

Enhanced images of inter object stretching and intra-object stretching can be combined together to reconstruct the final output enhanced image. Since the enhancement operation is directly applied to the object, the final enhanced image does not suffer from ringing, blocking, or other false contouring artifacts. Moreover,nose is not enhanced, since the uniform linear stretching is applied to homogeneous regions.

Step 10: Algorithm for getting the output enhanced image by using inter-object stretching and intra-object stretching images is given below. 
Algorithm: Enhance_Output $\left(\mathbf{I}_{a}^{\prime}, I_{\varepsilon}^{\prime}\right)$

Input: I' (Inter Object Stretched Image), I' (Intra Object Stretched Image)

Output: I' (Enhanced Image) \{

$\%$ Calculate the Region Mean values from the inter object stretched image

$\%$ Now perform the following operation for image enhancement

If (Region belongs to inter object stretched)

$\mathrm{I}=\mathrm{I}_{\mathrm{a}}+$ Inter Object Stretched Region mean

Else

$I^{\prime}=I_{a}$ - Inter Object Stretched Region mean

\}

\section{Comparison Of ObJect-Based Contrast Stretching With EXISTING MeTHODS}

We can compare different contrast enhancement techniques by using subjective and objective assessment. The subjective assessment is an estimation of quality where there is no pre established measure and is based only on the opinion of the evaluator. The quantitative measures are used for objective assessment. However we have used entropy as the quantitative measures. Entropy is a statistical measure of randomness that can be used to characterize the input image.

\subsection{Subjective Assessment On Images}

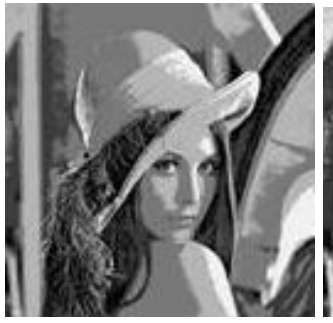

(a)

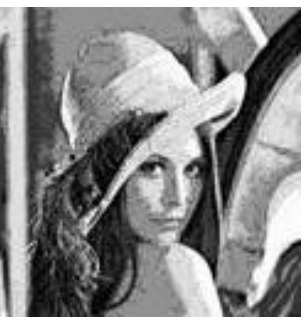

(b)

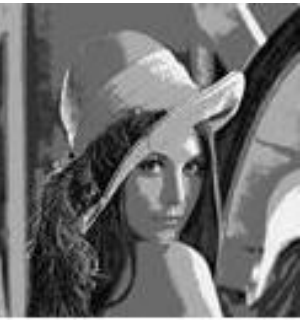

(c)

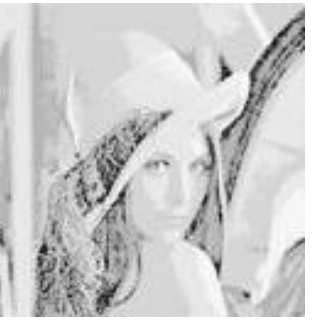

(d)

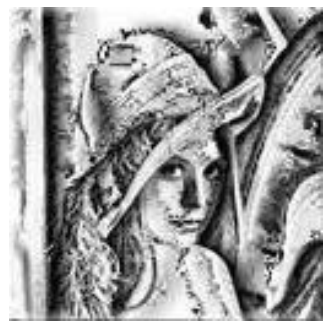

(e)

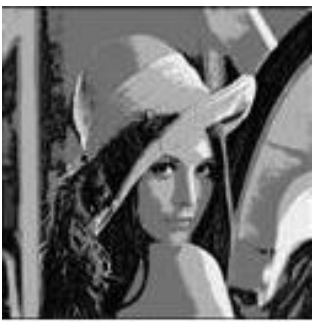

(f)

Fig 1: Results for Lena: (a) input Image (b) result of HE Method (c) result of power Law Transformation Method (d) result of Logarithmic Transformation (e) result of LHE Method (f) result after applying OBCS Method. 
Fig.1 shows the Lena image and their resultant contrast enhancement versions. The various methods are compared. Fig1 (a) demonstrates the original image. HE method enhances the contrast which results in over illumination of the image. This situation is observed in Fig1 (b). For Power Law Transformation, the foreground part of the image has improved but the hair of Lena is not much improved which is shown in the Fig1(c). For Logarithmic Transformation, the background as well as the foreground has over brightness look and is shown in Fig1 (d). An image with improved contrast and without natural look is shown in Fig1 (e) in the case of Local Histogram Equalization (LHE) method. The OBCS method preserves the brightness and also improves the contrast, but the portions became more dark which is observed in Fig1 (f).

Fig2 shows the Photographer image and their corresponding contrast enhancement versions. The various methods are compared. Fig2 (a) is the original image of photographer. HE method enhances the image but results in over illumination as shown in Fig2 (b). By applying Power Law Transformation method, the image is enhanced but a problem is observed in the background portion as in Fig2(c). In Logarithmic Transformation Method, the foreground image is brightened and background portion is damaged can be observed in Fig2 (d). In Local Histogram Equalization method, the contrast is improved but brightness is not preserved. Hence the output image does not give a natural look which is shown in Fig2 (e). The OBCS Method preserves the brightness and also improves the contrast of the image but the dark portion became more dark. It is observed in Fig2 (f).

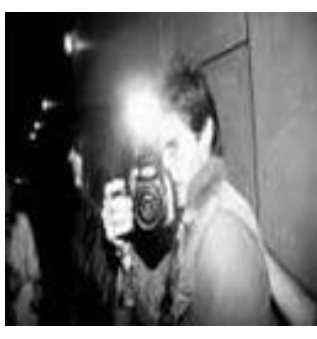

(a)

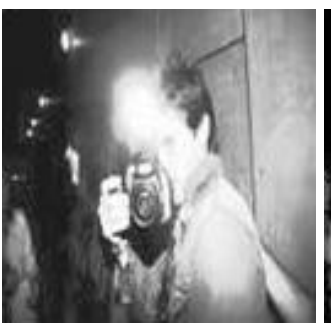

(b)

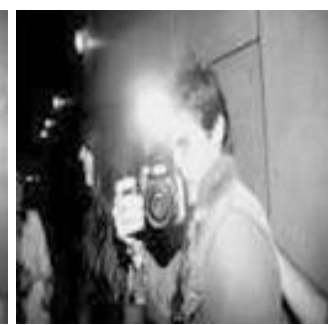

(d)

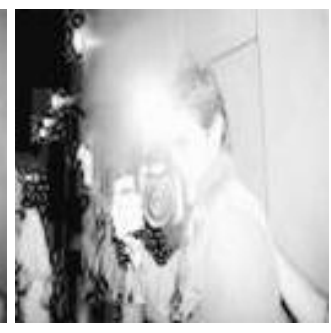

(d)

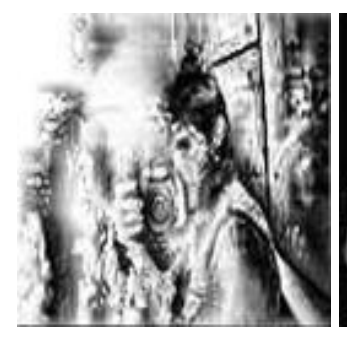

(e)

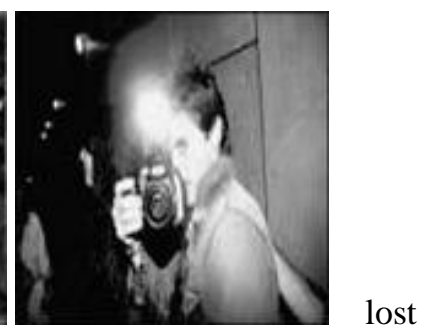

(f)

Fig 2: Results for Photographer: (a) Original input Image (b) result of HE Method (c) result of Power Law Transformation Method (d) result of Logarithmic Transformation (e) result after applying LHE Method (f) result after applying OBCS Method.

Fig3 demonstrates the original input image and their corresponding contrast enhancement versions. The different methods are compared. Fig3 (a) demonstrates the vegetables image. Over illumination and better utilization of dynamic range of the pixel values is observed in the case of HE method which is shown in Fig3 (b). By applying Power Law, the foreground brightness of the vegetables increased is shown in Fig3(c). Logarithmic Transformation increases both foreground and background parts of the image is shown in Fig3 (d).Contrast of the image is increased but the image missing its natural appearance by applying LHE method is shown in Fig 3(e). When 
compared to other methods OBCS preserves the brightness and enhancing the contrast of image, which is observed in fig3 (f).

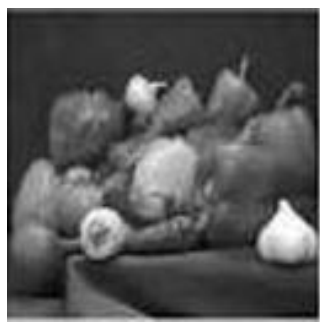

(a)

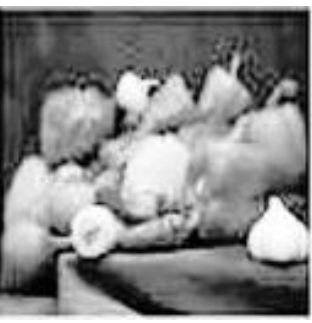

(b)

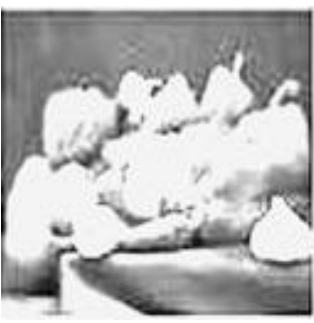

(c)

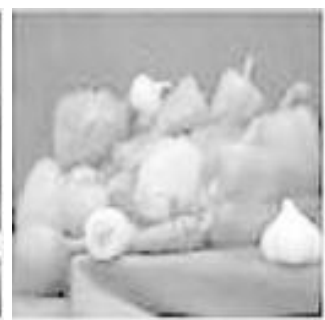

(d)

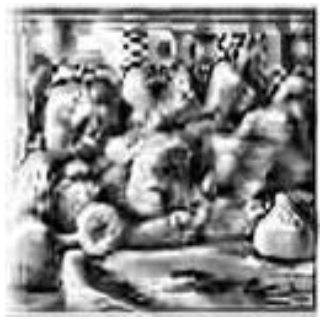

(e)

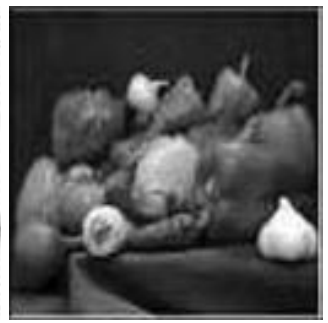

(f)

Fig 3: Results for vegetables: (a) Original input Image (b) result of Image after applying HE Method (c) result of Power Law Transformation Method (d) result after applying Logarithmic Transformation (e) result of LHE Method (f) result after applying OBCS Method

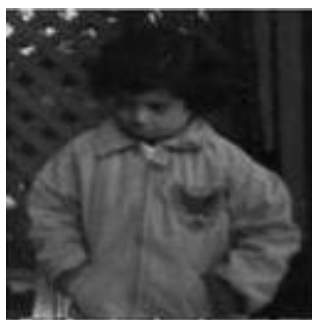

(a)

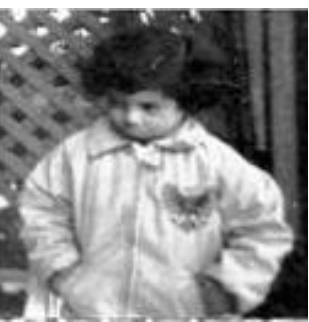

(b)

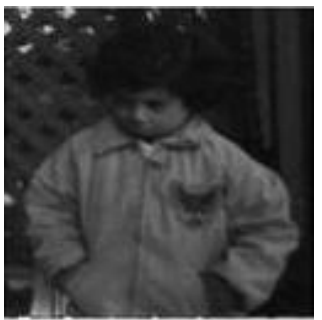

(c)

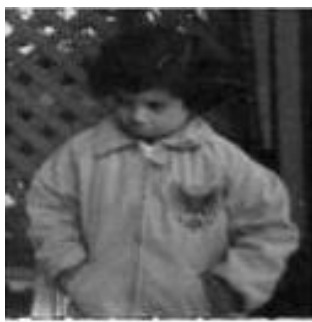

(d)

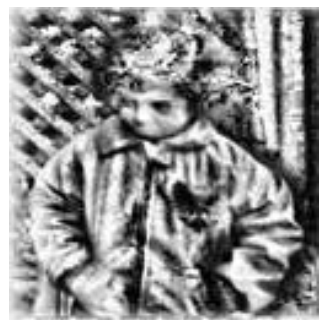

(e)

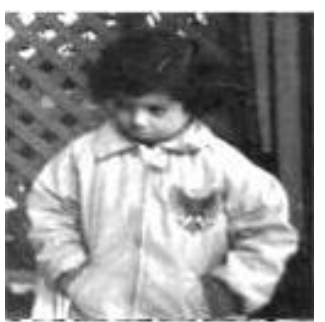

(f)

Fig 4: Results for Boy: (a) Original input Image (b) Enhanced Image of HE Method (c) result after applying Power Law Transformation Method (d) Enhanced Image of Logarithmic Transformation (e) result after applying LHE Method (f) Enhanced Image after applying OBCS Method. 
Fig4 demonstrates the original input image Boy and their corresponding contrast enhancement versions. The various methods are compared. Fig4 (a) is the original image with good contrast. HE method enhances the image but enhancement more than the required level which is shown in Fig4 (b). By applying Power Law Transformation method, the image is darkening as in Fig4(c). Logarithmic Transformation increases both the foreground and background which can be observed in Fig4 (d).Local Histogram Equalization increases the contrast but brightness is not preserved, hence the output image does not give a natural look which is shown in Fig4 (e). The OBCS Method preserves the brightness and also improves the contrast of the image. It is observed in Fig4 (f).

\subsection{Objective Assessment On Images}

Entropy is a statistical measure of randomness that can be used to characterize the input image. Entropy is defined as

$$
\text { Entropy }=\text { sum }(\mathrm{p} . * \log 2(\mathrm{p}))
$$

Where $\mathrm{p}$ contains the histogram counts returned from imhist.

The mean squared error of the estimator or predictor $\mathrm{T}(\mathrm{Y})$ for $\mathrm{U}$ is

$$
\operatorname{MSE}[\mathrm{T}(\mathrm{Y}) ; \mathrm{U}]=\mathrm{E}\left[(\mathrm{T}(\mathrm{Y})-\mathrm{U})^{2}\right]
$$

Table 1: Entropy Values of Different Images

\begin{tabular}{|c|c|c|c|c|}
\hline METHOD & LENA & Photographer & vegetables & BOY \\
\hline OBCS & 5.9767 & 6.8357 & 6.6142 & 6.1258 \\
\hline$H E$ & 6.3265 & 6.7479 & 6.5276 & 5.9967 \\
\hline$P L T$ & 0.1248 & 0.0000 & 0.0000 & 0.0188 \\
\hline$L T$ & 0.3581 & 0.0310 & 0.0011 & 0.0188 \\
\hline$L H E$ & 7.7450 & 7.7024 & 7.8382 & 7.8826 \\
\hline
\end{tabular}

Table 2: Mean Square Error Values of Different Images

\begin{tabular}{|c|c|c|c|c|}
\hline METHOD & LENA & Photographer & vegetables & BOY \\
\hline OBCS & 0.0030 & 0.0075 & 0.0099 & 0.0072 \\
\hline HE & 0.0148 & 0.0013 & 0.0030 & 0.0410 \\
\hline PLT & 0.0563 & 0.1130 & 0.1275 & 0.0320 \\
\hline LT & 0.1448 & 0.2734 & 0.3077 & 0.0886 \\
\hline LHE & 0.0450 & 0.0010 & 0.0009 & 0.0556 \\
\hline
\end{tabular}

\section{CONCLUSION}

The method is applied on various types of images. The assessment is performed by using both subjective and objective evaluation. Objective analysis has been performed using MSE and Entropy. It is seen that mean square error is less using OBCS method for all images. The subjective analysis shows that image has been improved in all the cases, but it can be observed that in the good contrast images the background is growing more darken which will be the future work of this research. 
International Journal of Advanced Information Technology (IJAIT) Vol. 4, No. 3, June 2014

\section{REFERENCES}

[1] J. M. Gauch, "Investigations of image contrast space defined by variations on histogram equalization," in Proc. CVGIP: Grap. Models Image Process., Jul. 1992, pp. 269-280.

[2] J. A. Stark, "Adaptive image contrast enhancement usign generalizations of histogram equalization," IEEE Trans. Image Process., vol. 9, no. 5, pp. 889-896, May 2000.

[3] Y.Wang, Q. Chen, and B. Zhang, "Image enhancement based on equal area dualistic sub-image histogram equalization method," IEEE Trans. Consum. Electron., vol. 45, no. 1, pp. 68-75, Feb. 1999.

[4] H. Zhu, F.H.Y. Chan, F.K. Lam, "Image contrast enhancement by constrained local histogram equalization," Computer Vision Image Understanding, vol. 73, no. 2, pp. 281-290, Feb. 1999.

[5] Joung-Youn Kim, Lee-Sup Kim, and Seung-Ho Hwang, "An Advanced Contrast Enhancement using Partially Overlapped Sub-block Histogram Equalization," IEEE Transactions on Circuits and Systems for Video Technology, vol. 11, no. 4, pp. 475-484, Apr. 2001.

[6] S. Mukhopadhyay and B. Chanda, "multiscale morphological approach to local contrast enhancement," Signal Processing, vol. 80, no. 4, pp. 685-696, 2000.

[7] R.C. Gonzalez, R.E. Woods, "Digital Image Processing," 3rd edition, Prentice Hall, 2008.

[8] Abdullah-Al-Wadud,M., Kabir, Md. Hasanul., Dewan, M. Ali Akber. and Chae, Oksam.(2007) "A DynamicHistogram Equalization for Image Contrast Enhancement" IEEE 2007

[9] N. Otsu, "A threshold selection method from grey-level histograms," IEEE Trans. on Systems, Man and Cyb., vol. 9, no. 1, pp. 41-47, 1979.

[10] Iyad Jafar ,and Hao Ying," Multilevel Component-Based Histogram Equalization for Enhancing the Quality of Grayscale Images”, IEEE EIT, pp. 563-568, 2007.

[11] K. Mardia and T. Hainsworth, "A spatial thresholding method for image segmentation," IEEE Trans. Pattern Anal. Machine Intell., vol. 10, pp.919-927, Nov. 1988.

[12] F. Meyer and S. Beucher, "Morphological segmentation," J. Vis. Commun. Image Represent., vol. 1, pp. 21-46, Sept. 1990.

[13] J. M. Gauch, "Image segmentation and analysis via multiscale gradient watershed hierarchies," IEEE Trans. Img. Proc., vol. 8, no. 1, Jan. 1999.

[14] L. Najman, "Geodesic saliency of watershed contours and hierarchical segmentation," IEEE Trans. Pattern Anal. Machine Intell., vol. 18, no. 12, pp. 1163-1173, Dec. 1996.

[15] K. Haris, S. N. Efstratiadis, N. Maglaveras, A. K. Katsaggelos, "Hybrid image segmentation using watersheds and fast region merging," IEEE Trans. Image Processing, pp. 1684-1699, vol. 7, no. 12, 1998.

[16] D. Shen, M. Huang, “A watershed-based image segmentation using JND property," IEEE Int. Conf. Acoust. Speech Signal Process Proc. 3:377-380, 2003.

[17] S. E. Hernandez, K. E. Barner, and Y. Yuan, "Region merging using homogeneity and edge integrity for watershed-based image segmentation," Opt. Eng., vol. 44, 2004.

[18] X. C. He, R. H. Y Chung and N. H. C. Yung "Watershed segmentation with boundary curvature ratio based merging criterion," Proceedings of IASTED International Conference on Signal and Image Processing, pp. 7-12, 2007.

[19] N. Pal, S. Pal, "A review on image segmentation techniques," Pattern Recognit., vol. 26, pp. 12771294, 1993.

[20] J. -L. Stark, F. Murtagh, E. J. Candes and D. L. Donoho, "Gray and color image contrast enhancement by curvelet transform," IEEE Transactions on Image Processing, vol. 12, no. 6, 2003.

[21] A. Khellaf, A. Beghdadi, H. Dupoisot, "Entropic contrast enhancement," IEEE Trans. Medical Imaging, vol. 10, no.4, 1991.

[22] Aditi Majumder, Sandra Irani, "Perception Based Contrast Enhancement of Images," ACM Transactions on Applied Perception, vol. 4, no. 3, Article 17, Jul. 2007.

[23] P. G. Barten, "Contrast sensitivity of the human eye and its effects on image quality," SPIE - The International Society for Optical Engineering, P.O. Box 10 Bellingham Washington, ISBN 0-81943496-5, 1999.

[24] Dr. A. Sri Krishna,G. Srinivasa Rao,M.Sravya "Contrast enhancement techniques using Histohram Equalization methods on color images with poor lightning" International Journal of Computer Science, Engineering and Applications (IJCSEA) Vol.3, No.4, August 2013 


\section{Authors}

G.Srinivasa Rao graduated in B.E(CSE) from Marathwada university, India in the year 1989, received masters degree M.Tech (CSE) from JNTU Hyderabad in the year 2008, M.S degree in software systems from Birla Institute of Technology and Science, pilani in 1996 and he is pursuing PhD from JNTUH, Hyderabad. He has 23 years of teaching Experience. Presently he is working as Associate Professor in the department of Information Technology, RVR \& JC College of Engineering, Guntur. His research interest includes Image and Signal Processing, algorithms and web technologies. He is a member of CSI.

Dr. A. Sri Krishna received the PhD degree from JNTUK, Kakinada in 2010, M.Tech degree in Computer Science from Jawaharlal Nehru Technological University (JNTU) in 2003, M.S degree in software systems from Birla Institute of Technology and Science, Pilani in 1994, AMIE degree in Electronics \& communication Engineering from Institution of Engineers, Kolkata in 1990. She has 23 years of teaching experience as Assistant Professor, Associate Professor, Professor and presently she is working as a Professor and Head, Dept of Information Technology at RVR\&JC College of Engineering, Guntur. She has published 15 papers in International/ National Journals

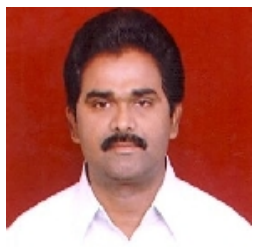
and Conferences. Her research interest includes Image Processing and Pattern Recognition. She is member of IE(I) and member of CSI.

Dr. S.Mahaboob Basha did his B.E.(Electronics) from Bangalore University, M.Tech. from JNTU Anantapur and Ph.D. from S.K.University, Anantapur. He worked in S.K.University in the Dept. Computer Science \& Technology and in Wipro Technologies, Bangalore. He has 21 years of teaching and 2 years of industrial experience. He has 6 years of research experience. He is an expert in java technologies and has delivered many guest lectures in various universities/colleges on Java, VC++, Software Engineering, Data Structures and DBMS. His areas of interest in research are: Image Compression, Image Filtering, Steganography, Network Security and Bio-Medical Engineering. He has published 10 papers in reputed national and international journals. He is the life-time member of ISTE

CH.Jeevan Prakash did his B.Tech Graduation in Information Technology, in RVR \& JC College of Engineering, Chowdavaram, Guntur Andhra Pradesh, under ANU university India. He has been actively participating and presenting papers in student technical Symposium seminars at National Level. Her area of interest includes Image Processing, web technologies.

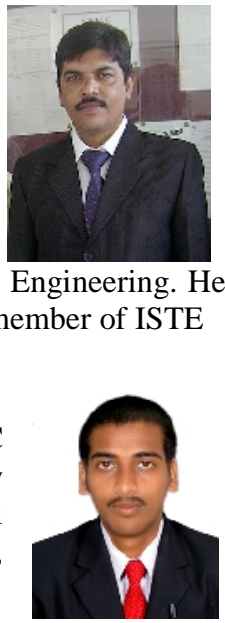

